\title{
Anharmonic Frequency Analysis
}

\author{
By A. K. Paul
}

\begin{abstract}
A new numerical method of frequency analysis is described, designed mainly to search for discrete frequencies in a time series. An integral transform is applied twice to the data for different reference times. A complex amplitude within a selected narrow frequency band is obtained for each transform. The frequency is then determined from the phase change of the complex amplitude over the difference of the two reference times. Very high precision is obtained, which is demonstrated in two examples.
\end{abstract}

1. Introduction. We consider a real time function $X(t)$ and assume that it can be described either by a set of discrete frequencies and their complex amplitudes (at a given reference time) or by a continuous complex amplitude density function over some frequency range or a combination of both. The term frequency analysis is used here for a process which determines the frequencies, amplitudes and phases of the spectral components of the time function.

There are three classical methods available which perform frequency analysis under specific conditions: the Fourier series, the Fourier integral and Prony's method. Each is restricted in its application, depending on the properties of the time function. Since all three methods are well known and described in standard textbooks, we will only repeat some of their essential properties.

(a) Fourier series. If $X(t)$ is periodic with a period $T$, it can be described by a constant term and a finite or infinite set of harmonic frequencies, where the basic frequency is equal to the reciprocal of the period $T$ and all other frequencies are integer multiples of the basic frequency. The complex amplitude is obtained by the well-known integral transform of $X(t)$ over the interval $T$. The integration extends only over the (finite) time interval $T$, since $X(t)$ is periodic, and no additional information is introduced into the process, if the integral transform were to be extended over several periods. On the other hand, when a time function $X(t)$ is given only for a time interval $T$ and this integral transform is applied, it is automatically assumed that the time function is periodic with the period $T$, and no additional information is obtained by an extrapolation. Therefore, for example, the Fourier series has no real application in prediction problems.

(b) Fourier integral. Here, a frequency continuum is provided for the analysis. The integral transform now determines the amplitude density as a function of frequency. The time function is aperiodic and must not contain discrete frequencies with finite amplitudes (equivalent to infinite amplitude density), otherwise the transform is not convergent. The integration has to be performed over the infinite time range from $-\infty$ to $+\infty$, unless the time function is zero outside a certain time

Received January 20, 1971.

AMS 1970 subject classifications. Primary 65T05, 42A76; Secondary 65H05.

$K e y$ words and phrases. Anharmonic frequency analysis, $P$-transform, Fourier-integral, Fourierseries, Prony method, tidal analysis, algebraic equations.

Copyright @ 1972, American Mathematical Society 
interval $T$. On the other hand, if $X(t)$ is only known within a time interval $T$ and this transform is applied, it is automatically assumed that $X(t)$ is zero outside the interval $T$. Again, nothing can be learned in this case by an extrapolation.

(c) Prony's method. This process [1], [2] is quite different from the two mentioned before. It is applicable if $X(t)$ contains only discrete frequencies and their number is finite. The method leads to an algebraic equation of degree $2 N$ with $N$ pairs of conjugate complex roots, if $N$ frequencies are present in the time function. The solution of the algebraic equation may result in real or complex frequencies. If a pair of roots is located inside the unit circle of the complex plane, the amplitude of the corresponding frequency is exponentially decreasing with time; if a pair of roots is located on the unit circle, the amplitude is constant, and if the roots are outside the unit circle, the amplitude is exponentially increasing with time. In contrast to the Fourier series, this method determines the inherent frequencies and their amplitudes rather than merely the amplitudes for a given set of frequencies; furthermore, the ratio of any pair of frequencies may be rational or irrational. Any small time interval is sufficient for this type of analysis, if $X(t)$ is given with sufficient precision within this interval.

Prony's method is only of practical value if the number of frequencies contained in $X(t)$ is relatively small, since it involves the inversion of a $2 N$ by $2 N$ matrix for the determination of the coefficients for the algebraic equation, the solution of the algebraic equation of degree $2 \mathrm{~N}$, and again a matrix inversion for the determination of the amplitudes.

We will show in the next chapter that, if we limit the problem of frequency analysis to real frequencies, it can be solved essentially by one process covering all the possibilities of the three methods discussed above.

2. The Basic Transform. We define a transform function $P$, depending on time $t$ and two parameters, the frequencies $f_{i}$ and $f_{l}$, by

$$
P\left(t ; f_{i}, f_{l}\right)=\frac{1}{i \pi t}\left(\exp \left(i 2 \pi f_{l} t\right)-\exp \left(i 2 \pi f_{i} t\right)\right),
$$

where we assume that

$$
\Delta f=f_{l}-f_{i}>0 .
$$

We see that $P$ is continuous, even for $t=0$, since it can be written

$$
P=\frac{2}{\pi t} \sin (\pi \Delta f t) \exp \left(i \pi\left(2 f_{i}+\Delta f\right) t\right),
$$

and therefore

$$
\lim _{t \rightarrow 0} P=2 \Delta f .
$$

We now define an integral transformation applied to a real function of time $X(t)$ by

$$
T\left(X ; f_{i}, f_{l}\right)=\int_{-\infty}^{+\infty} P\left(t ; f_{i}, f_{l}\right) X(t) d t,
$$

which we shall call the $P$-transform. 
We consider first the case where the transform is applied to

$$
X_{1}(t)=a \cos 2 \pi f_{1} t+b \sin 2 \pi f_{1} t,
$$

and we obtain (see Appendix A)

$$
\begin{aligned}
T\left(X_{1} ; f_{i}, f_{l}\right) & =0 & & \text { if } \quad f_{1}<f_{i}, \\
& =\frac{1}{2}(a+i b) & & \text { if } \quad f_{1}=f_{i}, \\
& =a+i b & & \text { if } \quad f_{i}<f_{1}<f_{l}, \\
& =\frac{1}{2}(a+i b) & & \text { if } \quad f_{1}=f_{l}, \\
& =0 & & \text { if } \quad f_{1}>f_{l},
\end{aligned}
$$

or, using the symbol $\Delta_{i, 1, l},(2.7)$ is conveniently written as

$$
T\left(X_{1} ; f_{i}, f_{l}\right)=\Delta_{i, 1, l}(a+i b) .
$$

Now, we apply the transform to $X_{N}(t)$ given by

$$
X_{N}(t)=\sum_{k=1}^{N}\left\{a_{k} \cos 2 \pi f_{k} t+b_{k} \sin 2 \pi f_{k} t\right\}, \quad f_{k+1}>f_{k},
$$

and obtain

$$
T\left(X_{N} ; f_{i}, f_{l}\right)=\sum_{k=1}^{N} \Delta_{i, k, l}\left(a_{k}+i b_{k}\right) .
$$

If $f_{i}$ and $f_{l}$ are properly chosen so that

$$
\Delta f<\min \left|f_{k+1}-f_{k}\right|, \quad k=1,2, \cdots, N-1,
$$

each frequency $f_{k}$ can be isolated. This means that, if the result of the transform is not zero, we obtain the complex amplitude of one frequency $f_{k}$ to

$$
a_{k}+i b_{k}=c_{k} \exp \left(i \varphi_{k}\right) \text {, }
$$

and the upper and lower limits $f_{l}$ and $f_{i}$ for the unknown frequency $f_{k}$ are known. It is important to notice that, if $f_{k}$ is equal to the upper or lower limit, we obtain only half of the actual amplitude, but still the correct phase $\varphi_{k}$.

We next apply the transform to $X_{N}(t+\Delta \tau)$ :

$$
\begin{aligned}
X_{N}(t+\Delta \tau) & =\sum_{k=1}^{N}\left\{a_{k} \cos 2 \pi f_{k}(t+\Delta \tau)+b_{k} \sin 2 \pi f_{k}(t+\Delta \tau)\right\} \\
& =\sum_{k=1}^{N}\left\{a_{k}^{\prime} \cos 2 \pi f_{k} t+b_{k}^{\prime} \sin 2 \pi f k t\right\}
\end{aligned}
$$

and obtain

$$
T^{\prime}=\sum_{k=1}^{N} \Delta_{i, k, l}\left(a_{k}^{\prime}+i b_{k}^{\prime}\right)
$$

As can be easily verified, we now have

$$
a_{k}^{\prime}+i b_{k}^{\prime}=c_{k} \exp \left(i \varphi_{k}^{\prime}\right),
$$


with

$$
\varphi_{k}^{\prime}=\varphi_{k}-2 \pi f_{k} \Delta \tau
$$

and we obtain the exact frequency

$$
f_{k}=\left(\varphi_{k}-\varphi_{k}^{\prime}\right) / 2 \pi \Delta \tau
$$

If $X(t)$ has a continuous spectrum given by

$$
X_{c}(t)=\int_{0}^{\infty}(A(\omega) \cos \omega t+B(\omega) \sin \omega t) d f,
$$

where $\omega=2 \pi f$, the transform leads to

$$
T\left(X_{c} ; f_{i}, f_{l}\right)=\int_{f_{i}}^{f_{l}}(A(\omega)+i B(\omega)) d f .
$$

Dividing (2.19) by $\Delta f=f_{l}-f_{i}$, we obtain the average complex amplitude density over the interval $f_{i} \leqq f \leqq f_{l}$ and it can be easily verified that in the limit as $\Delta f \rightarrow 0$ we obtain the Fourier integral. We see now that our transform can be considered as the integral of the Fourier integral over the frequency interval $\Delta f$. It may be worth mentioning here that, if $X(t)$ contains discrete frequencies, our transform converges, while the Fourier integral does not. In other words, discrete frequencies appear as integrable singularities in the Fourier integral.

3. The Transform of Discrete Data. In practice, we always have a finite time interval over which $X(t)$ has been observed. Even if recordings are taken continuously, only discrete data can be handled by a numerical method. We assume from here on that the data to be analyzed are available at equal time intervals $\Delta t$ for a certain period of time and that $\Delta t$ was properly chosen so that no aliasing can occur. We then approximate the integration by a summation and introduce at the same time normalized frequencies by

$$
\delta=\Delta f \cdot \Delta t
$$

and

$$
\beta=\left(f_{i}+\Delta f / 2\right) \Delta t, \quad 0 \leqq \beta \leqq 0.5 .
$$

The upper limit for $\beta$ is the normalized Nyquist frequency. (Later, we will formally extend the range of $\beta$ up to 1, so that the algorithm of the "Fast Fourier Transform" [3] can be used in the computation of the transform.) The transform of Section 2 then gives

$$
T_{K}(X ; \beta, \delta)=\frac{2}{\pi} \sum_{k=-K}^{+\pi} \frac{1}{k} \sin (\pi k \delta) \exp (i 2 \pi k \beta) X_{k},
$$

where the term for $k=0$ is $2 \delta X_{0}$, and $2 K+1$ data are used. Since the transform has to be applied again for a later data epoch, more than $2 K+1$ data must be available.

It should be mentioned that the transform (3.3) is an approximation of the original transform for two reasons:

(1) The integration is replaced by a summation.

(2) We have a finite series instead of an infinite one.

While the original transform produces a rectangular gate of unit height and width $\Delta f$ 
in the frequency domain, it is only approximated by the finite series (3.3). This affects mainly the frequency resolution.

If we search for discrete frequencies, the actual analysis consists of two steps. First, we scan systematically through the normalized frequency range, $0 \leqq \beta \leqq \frac{1}{2}$, in steps of $\delta$, repeat the transform for a later time and obtain a first estimate of the complex amplitude and the frequency in each band. Corrected values are obtained in a second step, if we set the gate individually for each frequency, where a large amplitude was obtained, so that the frequency is approximately in the center of the gate. We see that in this case the rectangular shape of the gate is not essential. If it is replaced by a cosine shape given by

$$
\begin{aligned}
g(\beta) & =0, & & \text { in } 0 \leqq \beta \leqq \beta_{c}-\delta, \\
& =\frac{1}{2}\left(1+\cos \pi \frac{\beta-\beta_{c}}{\delta}\right), & & \text { in } \beta_{c}-\delta \leqq \beta \leqq \beta_{c}+\delta, \\
& =0, & & \text { in } \beta_{c}+\delta \leqq \beta \leqq \frac{1}{2},
\end{aligned}
$$

where $\beta_{c}$ is the center of the gate, the convergence of the transform can be improved, For a cosine gate the transform is given by

$$
T C(X ; \beta, \delta)=\frac{1}{\pi} \sum_{k=-K}^{+K} \frac{\sin 2 \pi k \delta}{k\left(1-4 \delta^{2} k^{2}\right)} \exp (i 2 \pi k \beta) X_{k},
$$

where again the term for $k=0$ is $2 \delta X_{0}$.

The scanning process mentioned above is simplified if (3.5) is modified in the following way. We first introduce

$$
\beta=\beta_{0}+m \delta, \quad m=0,1, \cdots, M-1 \text { and } \delta=1 / M,
$$

where $\beta_{0}$ may be any fraction of $\delta$. We then obtain

$$
T_{m}=\frac{1}{\pi} \sum_{-K}^{+K} \frac{\sin 2 \pi k \delta}{k\left(1-4 \delta^{2} k^{2}\right)} \exp \left(i 2 \pi k \beta_{0}\right) X_{k} \exp \left(i 2 \pi \frac{k m}{M}\right)
$$

The term $\beta_{0}$ permits us to shift all the gates by a fraction of the "bandwidth" $\delta$, if this is desirable.

If we now set

$$
\begin{gathered}
Y_{k}=\frac{1}{\pi} \frac{\sin 2 \pi k \delta}{k\left(1-4 \delta^{2} k^{2}\right)} X_{k} \exp \left(i 2 \pi k \beta_{0}\right), \quad-K \leqq k \leqq-1,1 \leqq k \leqq K, \\
Y_{0}=2 \delta X_{0},
\end{gathered}
$$

we have

$$
T_{m}=\sum_{-K}^{+K} Y_{k} \exp (i 2 \pi k m / M)
$$

We must point out that $M$ and $K$ are mutually independent; $M$ determines the bandwidth, while $2 K+1$ is the number of data to be transformed. Usually, $M$ is smaller than $K$ as will be discussed later.

Since the exponential function in (3.10) is periodic in $k$ with the period $M$, we set

$$
k=r \cdot M+s, \quad s=0,1, \cdots, M-1, L^{-} \leqq r \leqq L^{+},
$$


where $L^{-}$and $L^{+}$are integers defined by

$$
-K / M-1<L^{-} \leqq-K / M
$$

and

$$
K / M-1<L^{+} \leqq K / M .
$$

Introducing now $Z$, by

$$
Z_{.}=\sum_{r=L^{-}}^{L^{+}} Y_{r \cdot M+\cdots}
$$

we obtain

$$
T_{m}=\sum_{s=0}^{M-1} Z_{s} \cdot \exp \left(i 2 \pi \frac{s \cdot m}{M}\right)
$$

If $M$ is properly chosen, e.g., $M=2^{p}$, where $p$ is an integer, the algorithm of the "Fast Fourier Transform" [3] can be used to perform the transform (3.14).

If $X(t)$ is represented by an amplitude density function, the first step, the scanning process, is usually sufficient. It is easy to see how a continuous spectrum can be distinguished from a line spectrum. If sufficient resolution is obtainable, the amplitude of a discrete frequency does not change with decreasing bandwidth, while for a continuous spectrum the ratio of amplitude and bandwidth should remain constant if the bandwidth is reduced.

4. Examples. As a test, the method was applied to tidal data. The frequencies of the tides are very well known from astronomical observations. Two years (19511952) of bi-hourly measurements of tides at San Francisco were analyzed; the results are listed in Table 1. The first column shows the theoretical frequencies in degrees per hour as listed by Schureman [4]. The second and third columns show the results of our analysis, the amplitudes and frequencies, respectively. The frequency differences between the theoretical values and those determined in the analysis are listed in columns 4 and 5 in different units, degrees per hour and cycles per year. The largest difference found is 0.05 cycles per year. For comparison, the difference between two consecutive frequencies provided by a Fourier series analysis of two years of data amounts to 0.5 cycles per year, ten times larger than the largest difference found in the analysis above.

The second example is directly related to Prony's method. There, a polynomial equation is derived from the time series, and the roots of the polynomial equation determine the frequencies. By the inverse process, a time series can be produced from a polynomial and the frequency analysis of this time series determines the roots of the polynomial equation, provided that those roots are located on the unit circle of the complex plane or that the polynomial can be properly transformed. In other words, if it is known that all the roots of a polynomial equation are located on a curve which can be mapped by a proper transform onto the unit circle, then the roots of this polynomial equation can be found by the frequency analysis of the corresponding time series. 
TABLE 1

Analysis of Tides, San Francisco 1951-1952

\begin{tabular}{|c|c|c|c|c|}
\hline \multirow{3}{*}{$\begin{array}{c}\text { Table } \\
\text { Frequency } \\
\text { Deg/Hour }\end{array}$} & \multicolumn{2}{|c|}{ Results } & \multirow{2}{*}{\multicolumn{2}{|c|}{$\begin{array}{l}\text { Frequency } \\
\text { Differences }\end{array}$}} \\
\hline & Ampl. & Frequency & & \\
\hline & Feet & Deg/Hour & Deg/Hour & C/Year \\
\hline 1.0980331 & 0.058 & 1.0994840 & 0.001451 & 0.03534 \\
\hline 13.3986609 & 0.156 & 13.3984707 & 0.000190 & 0.00463 \\
\hline 13.9430356 & 0.900 & 13.9426864 & 0.000349 & 0.00850 \\
\hline 14.9589314 & 0.380 & 14.9591985 & 0.000267 & 0.00650 \\
\hline 15.0410686 & 1.344 & 15.0412392 & 0.000171 & 0.00415 \\
\hline 15.5854433 & 0.089 & 15.5860805 & 0.000637 & 0.01552 \\
\hline 16.1391017 & 0.066 & 16.1406875 & 0.001586 & 0.03861 \\
\hline 27.8953548 & 0.043 & 27.8945316 & 0.000823 & 0.02004 \\
\hline 27.9682084 & 0.029 & 27.9662659 & 0.001943 & 0.04730 \\
\hline 28.4397295 & 0.378 & 28.4396179 & 0.000112 & 0.00271 \\
\hline 28.5125831 & 0.074 & 28.5133208 & 0.000738 & 0.01796 \\
\hline 28.9841042 & 1.778 & 28.9840174 & 0.000087 & 0.00211 \\
\hline 29.5284789 & 0.053 & 29.5288241 & 0.000345 & 0.00841 \\
\hline 30.0000000 & 0.431 & 29.9997015 & 0.000298 & 0.00727 \\
\hline 30.0821373 & 0.159 & 30.0824543 & 0.000317 & 0.00772 \\
\hline 30.6265120 & 0.016 & 30.6282672 & 0.001755 & 0.04274 \\
\hline 42.9271398 & 0.063 & 42.9257891 & 0.001351 & 0.03289 \\
\hline 44.0251729 & 0.081 & 44.0251719 & 0.000001 & 0.00002 \\
\hline 45.0410686 & 0.014 & 45.0415260 & 0.000457 & 0.01114 \\
\hline 45.1232058 & 0.014 & 45.1232532 & 0.000047 & 0.00115 \\
\hline 57.4238337 & 0.029 & 57.4242372 & 0.000403 & 0.00983 \\
\hline 57.9682084 & 0.086 & 57.9683389 & 0.000130 & 0.00318 \\
\hline 58.9841042 & 0.039 & 58.9834004 & 0.000704 & 0.01714 \\
\hline 59.0662415 & 0.036 & 59.0663642 & 0.000123 & 0.00299 \\
\hline 71.3668693 & 0.013 & 71.3671729 & 0.000303 & 0.00739 \\
\hline 71.9112440 & 0.022 & 71.9118317 & 0.000588 & 0.01431 \\
\hline 73.0092770 & 0.023 & 73.0072166 & 0.002060 & 0.05017 \\
\hline 74.0251728 & 0.009 & 74.0247541 & 0.000419 & 0.01020 \\
\hline
\end{tabular}

The roots of the Legendre polynomials $L_{n}(x)$, for example, are located on the real axis between +1 and -1 , symmetrically around zero. Introducing

$$
x=i(1-y) /(1+y),
$$

$L_{n}(x)$ is now transformed into a polynomial $M_{n}(y)$ and its roots appear now as conjugate complex pairs on the unit circle. We applied this method to the Legendre polynomial of 16 th degree. The resulting polynomial $M_{16}(y)$ was normalized so that the coefficient of the highest power was unity. The time series was produced using the recurrence relation 


$$
X_{n}=-\sum_{i=0}^{15} c_{i} \cdot X_{n-16+i}
$$

starting with 16 arbitrarily chosen numbers $X_{0}, X_{1}, \cdots, X_{15}$. A total of 2200 data were produced this way. This time series was then analyzed. The results are given in Table 2 showing the "frequencies", the roots derived from the frequencies and

TABLE 2

\begin{tabular}{cccr}
\hline $\begin{array}{c}\text { Frequency } \\
\text { Calculated }\end{array}$ & $\begin{array}{c}\text { Root } \\
\text { Calculated }\end{array}$ & $\begin{array}{c}\text { Root } \\
\text { Exact }\end{array}$ & \multicolumn{1}{c}{$\begin{array}{c}\text { Root } \\
\text { Difference }\end{array}$} \\
\hline 0.030152905 & 0.095012511 & 0.095012510 & 0.000000001 \\
0.087374490 & 0.281603551 & 0.281603551 & 0.000000000 \\
0.136714302 & 0.458016778 & 0.458016778 & 0.000000000 \\
0.176171855 & 0.617876244 & 0.617876244 & -0.000000000 \\
0.205930889 & 0.755404410 & 0.755404408 & 0.000000002 \\
0.227113810 & 0.865631202 & 0.865631202 & 0.000000000 \\
0.240929873 & 0.944575025 & 0.944575023 & 0.000000002 \\
0.248304135 & 0.989400934 & 0.989400935 & -0.000000001 \\
\hline
\end{tabular}

the roots listed in Tables of Functions and Zeros of Functions [5] in the columns 1,2 , and 3, respectively. The differences between columns 2 and 3 are listed in column 4 , the maximum error amounts to $2 \times 10^{-9}$.

5. Discussion of the Numerical Application. Some comments on practical aspects may explain more details of the method. At the same time, we will attempt to demonstrate that the method is very flexible, but also quite complex in the sense that questions regarding the best choice of bandwidth $\delta$, time interval $\Delta \tau$ between two transforms, and error estimates of amplitude, phase and frequency, cannot easily be answered. Usually, those questions must be studied individually for a given time series, and the answers will depend mainly on the length of the time series, the frequency distribution in the spectrum, the amplitude ratio of neighboring frequencies and the noise level. Therefore, the following discussion will be restricted to some general considerations.

We assume that $N_{t}$ is the total number of data points available and use, instead of the time interval $\Delta \tau$ between two transforms, the corresponding number of data points $\Delta K$ given by

$$
\Delta K=\Delta \tau / \Delta t
$$

Since we need $2 K+1$ data points for one transform, we have the relation

$$
2 K+1+\Delta K \leqq N_{t} .
$$

As mentioned before, the original discrete transform is an infinite series, and we expect that the approximation by a finite series will become better, the larger the number $K$. More careful investigation of (3.3) and (3.5) shows that the convergence depends in first order on the product $K \cdot \delta$. This, together with (5.2), means that a 
compromise must be sought between the convergence, the interval between two transforms and the bandwidth.

With the finite series (3.3) or (3.5), we obtain an approximation of the desired shape of the gate, but also "sidelobes" in the remaining frequency range. The magnitude of those sidelobes decreases with increasing $K \cdot \delta$ and also with increasing frequency difference from the gate. Those sidelobes can introduce interference errors, for example, if the gate is set for a frequency with a relatively small amplitude and a strong line is in one of the neighboring sidelobes. (In many cases, the method permits us to eliminate this interference to a high degree by changing the bandwidth or shifting the gate, so that a zero between two sidelobes falls on such a strong line, if its frequency was already determined.) An estimate of the interference error may be difficult, especially, if several frequencies with different amplitudes are close together.

Generally, a wide bandwidth is desirable to keep interference low; on the other hand, the bandwidth must be small enough to separate the frequencies present in the record. This holds first of all for the scanning process when we start the analysis of a time series and nothing is known about its frequency distribution. Here, a reasonable compromise can be made by using a minimum bandwidth defined by the relation

$$
K \cdot \delta_{\min } \approx 1
$$

While the errors of amplitude and phase depend essentially on $K \cdot \delta$ and on the noise level, the error of the frequencies depends largely on $\Delta K$. If we can assume that the magnitude of the phase error remains within certain limits as a function of time, then the error of the frequency will decrease with increasing $\Delta K$. If $\Delta K$ corresponds to many periods of a given frequency, the transform may be repeated several times in smaller steps in order to avoid possible uncertainties about the number of full phase rotations. Repeating the transform several times also permits us to test the stability of a line in time. A systematic variation of the amplitude, for instance, may indicate that the gate includes more than one line and the spectrum is not sufficiently resolved with the bandwidth used.

The choice of the parameters $\delta, K$ and $\Delta K$ also depends to some extent on the problem to be solved. The first example given above, the analysis of tides, was used as a test on the frequencies. Since the frequencies are known from astronomical observations with high accuracy, the main emphasis of tidal analysis is usually on an accurate determination of amplitude and phase for each frequency, e.g., for prediction purposes. Therefore, $\Delta K$ can be small to minimize interference errors in phase and amplitude. In the second example, we have the opposite situation. Amplitude and phase are of no interest; only the accuracy of the frequencies is important, which requires a relatively large $\Delta K$.

In conclusion, it should be mentioned that there is no need to have the length of the time series equal to a joint multiple of the periods present. Some minimum length is required only for the resolution.

Appendix. The proof for (2.7) and (2.14) can be obtained in the following way. If we introduce (2.6) into (2.5), we have 
(A.1)

$$
\begin{aligned}
T= & \int_{-\infty}^{+\infty} \frac{1}{i \pi t}\left[\exp \left(i 2 \pi f_{l} t\right)-\exp \left(i 2 \pi f_{i} t\right)\right]\left(a \cos 2 \pi f_{1} t+b \sin 2 \pi f_{1} t\right) d t \\
= & \frac{(a-i b)}{\pi} \int_{0}^{\infty} \frac{1}{t}\left(\sin 2 \pi\left(f_{l}+f_{1}\right) t-\sin 2 \pi\left(f_{i}+f_{1}\right) t\right) d t \\
& +\frac{a+i b}{\pi} \int_{0}^{\infty} \frac{1}{t}\left(\sin 2 \pi\left(f_{l}-f_{1}\right) t-\sin 2 \pi\left(f_{i}-f_{1}\right) t\right) d t
\end{aligned}
$$

The first integral in (A.2) is always zero. For the second integral we have to distinguish the five possibilities of (2.7). We set

$$
2 \pi\left(f_{l}-f_{1}\right) t=y
$$

and

$$
2 \pi\left(f_{i}-f_{1}\right) t=x .
$$

For $f_{1}<f_{i}<f_{l}$, we have $y>0$ and $x>0$, and the second integral is again zero: For $f_{1}=f_{i}<f_{l}$, we have $y>0$ and $x=0$ and therefore

$$
T=\frac{a+i b}{\pi} \int_{0}^{\infty} \frac{\sin y}{y} d y=\frac{a+i b}{2} .
$$

For $f_{i}<f_{1}<f_{l}$, we have $y>0$ and $x<0$. Replacing $x$ by $-x$, we obtain

$$
T=\frac{a+i b}{\pi}\left(\int_{0}^{\infty} \frac{\sin y}{y} d y+\int_{0}^{\infty} \frac{\sin x}{x} d x\right)=a+i b .
$$

For $f_{i}<f_{l}=f_{1}$, we have $y=0$ and $x<0$. Again replacing $x$ by $-x$, we have

$$
T=\frac{a+i b}{\pi} \int_{0}^{\infty} \frac{\sin x}{x} d x=\frac{a+i b}{2},
$$

and finally, for $f_{i}<f_{l}<f_{1}$, we have $y<0$ and $x<0$ and the integral is zero.

For a continuous spectrum, we introduce (2.18) into (2.5) and obtain

$$
\begin{aligned}
T\left(X_{c}\right)= & \int_{-\infty}^{+\infty} \frac{1}{i \pi t}\left[\exp \left(i 2 \pi f_{l} t\right)-\exp \left(i 2 \pi f_{i} t\right)\right] \\
& \cdot \int_{0}^{\infty}(A(\omega) \cos \omega t+B(\omega) \sin \omega t) d \omega d t
\end{aligned}
$$

By changing the order of integration, we have

$$
\begin{aligned}
T\left(X_{c}\right)= & \int_{0}^{\infty} A(\omega) \int_{-\infty}^{+\infty} \frac{1}{i \pi t}\left[\exp \left(i 2 \pi f_{l} t\right)-\exp \left(i 2 \pi f_{i} t\right)\right] \cos \omega t d t d \omega \\
& +\int_{0}^{\infty} B(\omega) \int_{-\infty}^{+\infty} \frac{1}{i \pi t}\left[\exp \left(i 2 \pi f_{l} t\right)-\exp \left(i 2 \pi f_{i} t\right)\right] \sin \omega t d t d \omega .
\end{aligned}
$$

Now using the results of the first part of this Appendix, we see that we obtain (2.19).

U. S. Department of Commerce

National Oceanic and Atmospheric Administration

Environmental Research Laboratories

Boulder, Colorado 80302 
1. E. Whittaker \& G. Robinson, The Calculus of Observations, Blackie and Son, London and Glasgow, 1948, pp. 369 ff.

2. F. A. WILlers, Practical Analysis. Graphical and Numerical Methods, de Gruyter, Berlin, 1928; English transl., Dover, New York, 1948. MR 10, 404.

3. J. W. COOLEY \& J. W. TUKEY, "An algorithm for the machine calculation of complex Fourier series," Math. Comp., v. 19, 1965, pp. 297-301. MR 31 \#2843.

4. P. Schureman, Manual of Harmonic Analysis and Prediction of Tides, U.S. Government Printing Office, Washington, D. C., 1941.

5. Tables of Functions and Zeros of Functions, Nat. Bur. Standards Appl. Math. Series 37, U. S. Government Printing Office, Washington, D. C., 1954. 